\title{
The spatio-temporal dynamics of neutral genetic diversity
}

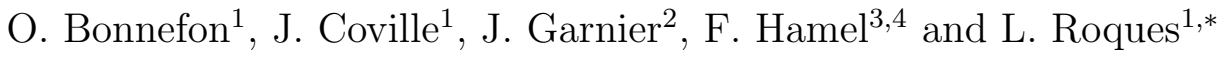 \\ ${ }^{1}$ UR 546 Biostatistique et Processus Spatiaux, INRA, 84000 Avignon, France \\ ${ }^{2}$ LAMA, UMR 5127 CNRS, Université de Savoie, Campus Scientifique, \\ 73376 Le Bourget-du-Lac Cedex, France \\ 3 Aix Marseille Université, CNRS, Centrale Marseille, \\ I2M, UMR 7373, 13453 Marseille, France \\ ${ }^{4}$ Institut Universitaire de France \\ * Corresponding author: lionel.roques@avignon.inra.fr
}

\begin{abstract}
The notions of pulled and pushed solutions of reaction-dispersal equations introduced by Garnier et al. (2012) and Roques et al. (2012) are based on a decomposition of the solutions into several components. In the framework of population dynamics, this decomposition is related to the spatio-temporal evolution of the genetic structure of a population. The pulled solutions describe a rapid erosion of neutral genetic diversity, while the pushed solutions are associated with a maintenance of diversity. This paper is a survey of the most recent applications of these notions to several standard models of population dynamics, including reaction-diffusion equations and systems and integro-differential equations. We describe several counterintuitive results, where unfavorable factors for the persistence and spreading of a population tend to promote diversity in this population. In particular, we show that the Allee effect, the existence of a competitor species, as well as the presence of climate constraints are factors which can promote diversity during a colonization. We also show that long distance dispersal events lead to a higher diversity, whereas the existence of a nonreproductive juvenile stage does not affect the neutral diversity in a range-expanding population.
\end{abstract}

Keywords. reaction-diffusion; genetic diversity; pushed and pulled solutions; traveling waves; long distance dispersal; Allee effect; juvenile stage; climate change; gene surfing;

\section{Introduction and definitions}

New mathematical tools have been introduced in the recent papers of Garnier et al. (2012) and Roques et al. (2012) to study the spatio-temporal dynamics of the neutral genetic structure in a range-expanding population. The framework proposed in these papers, inspired from a simulation study of Hallatschek and Nelson (2008), can be applied to a wide range of reactiondispersion equations. In this paper, we chiefly consider general models of reaction-dispersion where the unknown quantity is a population density $u(t, x) \in \mathbb{R}$ of genes or haploid individuals (a reaction-diffusion system involving two species will also be investigated). The density evolves in space and time under the intertwined effect of two factors: the dispersion, represented by a linear operator $\mathcal{D}$, and the reproduction (birth and death), embodied in the operator $\mathcal{F}$. 
The general form of these models is:

$$
\partial_{t} u(t, x)=\mathcal{D}[u](t, x)+\mathcal{F}[u](t, x), t>0, x \in \mathbb{R} .
$$

The operators $\mathcal{D}$ and $\mathcal{F}$ act on a function space. Thus $\mathcal{D}[u](t, x)$ and $\mathcal{F}[u](t, x)$ may depend on the population density $u$ at other points in time and space than the point $(t, x)$, meaning in particular that the operator can be nonlocal.

Inside dynamics of the solution. The notion of inside dynamics of solutions of reactiondispersion equations has been recently introduced in (Garnier et al., 2012; Roques et al., 2012). They assume that the population $u$ is made of several neutral components $\mu^{i} \geq 0(i \in I \subset \mathbb{N})$. This means that the population density $u(t, x)>0$ satisfying (1.1) is equal to the sum of the component densities:

$$
u(t, x)=\sum_{i \in I} \mu^{i}(t, x) .
$$

Since the components are neutral, the genes (or the individuals) in each component only differ by their position and their allele (or their label), while their dispersal and growth abilities are the same as the population $u$, in the sense that the density $\mu^{i}$ of each component satisfies an equation of the form:

$$
\left\{\begin{aligned}
\partial_{t} \mu^{i}(t, x) & =\mathcal{D}\left[\mu^{i}\right](t, x)+\frac{\mu^{i}}{u} \mathcal{F}[u](t, x), & & t>0, x \in \mathbb{R}, \\
\mu^{i}(0, x) & =\mu_{0}^{i}(x), & & x \in \mathbb{R},
\end{aligned}\right.
$$

where the components $\mu^{i}$ are such that

$$
u(0, x)=\sum_{i \in I} \mu_{0}^{i}(x), \quad x \in \mathbb{R} .
$$

The equation (1.3) is linear with respect to $\mu^{i}$. Therefore, the function $w=\sum_{i \in I} \mu^{i}(t, x)$ is a solution of the equation:

$$
\partial_{t} w(t, x)=\mathcal{D}[w](t, x)+\frac{w}{u} \mathcal{F}[u](t, x), t>0, x \in \mathbb{R} .
$$

In all the cases investigated in this paper, the assumptions on $\mathcal{D}$ guarantee the uniqueness of the solution $w$ of (1.4). As $u$ solves this equation with $u(0, \cdot)=w(0, \cdot)$, the fundamental property (1.2) is fulfilled at every $t>0$.

Note that for diploid populations following a reaction-diffusion equation with malthusian growth, the system (1.3), which governs the dynamics of the allelic densities, can also be derived from a weighted sum of the equations governing the genotype densities, such as those given by Aronson and Weinberger (1975).

The neutral assumption reduces the analysis of the card $(I)+$ 1-dimensional system (1.1)(1.3) to the analysis of a system of two equations. Namely, each component $\mu$ is given as the solution of:

$$
\left\{\begin{array}{lll}
\partial_{t} u(t, x)=\mathcal{D}[u](t, x)+\mathcal{F}[u](t, x), & & t>0, x \in \mathbb{R}, \\
\partial_{t} \mu(t, x)=\mathcal{D}[\mu](t, x)+\frac{\mu}{u} \mathcal{F}[u](t, x), & t>0, x \in \mathbb{R},
\end{array}\right.
$$

with initial conditions $\mu(0, x)=\mu_{0}(x)$ and $u(0, x)=u_{0}(x)$, such that $0 \leq \mu_{0}(x) \leq u_{0}(x)$ for $x \in \mathbb{R}$. 
Traveling wave solutions. For a large class of dispersal operators $\mathcal{D}$ and growth functions $\mathcal{F}$, the equation (1.1) admits traveling wave solutions. These particular solutions keep a constant speed $c$ and a constant profile $U$, that is $u(t, x)=U(x-c t)$. Typically, they describe the invasion of the stationary state 0 by a positive stationary state, say 1 . There are many references about the existence, uniqueness, stability and spreading properties of these waves, for reactiondiffusion, delay-differential and integro-differential equations. Relevant references will be given in the sections below. These studies had numerous implications in population dynamics (Fife, 1979; Murray, 2002; Shigesada and Kawasaki, 1997). However, despite important consequences in population genetics, and until very recently, the inside dynamics of the waves had not been investigated.

Pulled and pushed solutions. The notions of pulled and pushed waves have been introduced by Stokes (1976); Rothe (1981) for traveling wave solutions of scalar reaction-diffusion equations $\partial_{t} u=d \partial_{x x} u+f(u)$ with monostable growth term $f$. These definitions were heuristically related to the idea of an inside dynamics of the waves. For this equation, when $f>0$ in $(0,1), f^{\prime}(0)>0$ and $f(0)=f(1)=0$, it is known that there exists a minimal speed $c^{*}$ such that positive traveling waves with speed $c$ exist if and only if $c>c^{*}$. Stokes's notions of pulled and pushed waves are based on the linear determinacy of the minimal speed $c^{*}$ of propagation of traveling waves (see Hadeler and Rothe, 1975). If $c^{*}$ is linearly determined, that is if $c^{*}=2 \sqrt{d f^{\prime}(0)}$, then the wave is said to be pulled since the speed is determined by the growth of the pioneering individuals at the leading edge. Otherwise, the critical wave with speed $c^{*}>2 \sqrt{d f^{\prime}(0)}$ is said to be pushed by its nonlinear part.

Based on the above-described notions of inside dynamics, the pulled/pushed terminology has been redefined and extended in the works of Garnier et al. (2012) and Roques et al. (2012), leading to the following definitions:

Definition 1.1 (Pulled wave). A traveling wave $u(t, x)=U(x-c t)$ is said to be a pulled wave if, for any component $\mu^{i}$ satisfying (1.3), $0 \leq \mu_{0}^{i} \leq U$ and $\mu_{0}^{i}(x)=0$ for large $x$, there holds

$$
\mu^{i}(t, x+c t) \rightarrow 0 \text { as } t \rightarrow+\infty \text {, uniformly on compact sets. }
$$

Definition 1.2 (Pushed wave). A traveling wave $u(t, x)=U(x-c t)$ is said to be a pushed wave if, for any component $\mu^{i}$ satisfying (1.3), $0 \leq \mu_{0}^{i} \leq U$ and $\mu_{0}^{i} \not \equiv 0$, there exists $M>0$ such that

$$
\limsup _{t \rightarrow+\infty} \sup _{x \in[-M, M]} \mu^{i}(t, x+c t)>0 .
$$

We first focus on a pulled wave $U$. Let us consider the (right) component $\mu^{r}$, which satisfies at $t=0, \mu_{0}^{r}=U \cdot \mathbb{1}_{[\alpha,+\infty)}$, where $\mathbb{1}_{[\alpha,+\infty)}$ denotes the indicator function of the interval $[\alpha,+\infty)$, for some $\alpha \in \mathbb{R}$. The component $\mu^{l}$ corresponding to the remaining part of the wave satisfies at $t=0 \mu_{0}^{l}=U \cdot \mathbb{1}_{(-\infty, \alpha)}$. Therefore, $\mu^{l}$ converges to 0 in the moving frame with speed $c$ from definition 1.1. Since for all $t>0$ and $x \in \mathbb{R}$

$$
u(t, x+c t)=U(x)=\mu^{l}(t, x+c t)+\mu^{r}(t, x+c t),
$$

it follows that $\mu^{r}(t, x+c t)$ converges to $U(x)$ as $t \rightarrow \infty$ in the moving frame. Thus, the wave $U$ tends to be made of the rightmost component only. In other words, it is pulled by the component at the leading edge of the front, which indicates a strong erosion of the genetic diversity. 
On the other hand, in a pushed wave $U$, any component $\mu$ propagates with the same speed $c$ as the total wave $U(x-c t)$. In other words, the wave is pushed by all its components. This shows that the initial diversity in the pushed front is preserved during the colonization.

\section{The classical Fisher-KPP equation}

In that case, the dispersal operator $\mathcal{D}$ is the Laplace diffusion operator:

$$
\mathcal{D}=d \partial_{x x}
$$

for some positive diffusion coefficient $d>0$, and the growth functional $\mathcal{F}[u](t, x)$ is "local" in the sense that it only depends on the value of $u(t, x)$. In other words, $u$ satisfies the following equation:

$$
\partial_{t} u=d \partial_{x x} u+f(u), t>0, x \in \mathbb{R} .
$$

Furthermore, we assume that $f$ is the classical Fisher-KPP nonlinearity (Fisher, 1937; Kolmogorov et al., 1937), that is:

$$
f(u)=u(1-u) .
$$

This model, $\partial_{t} u=d \partial_{x x} u+u(1-u)$, is probably the simplest reaction-diffusion model for the dynamics of a population in expansion. Thus, the results presented in this section will serve as a reference for the other models presented in the next sections.

Equation (2.7) with the reaction term (2.8) admits positive traveling wave solutions $u(t, x)=$ $U(x-c t)$ such that $U(-\infty)=1$ and $U(+\infty)=0$ if and only if $c \geq 2 \sqrt{d}$ (Aronson and Weinberger, 1975; Fisher, 1937; Kolmogorov et al., 1937). Moreover, these waves are attractive in the sense that any solution $u$ of (2.7) starting from a step-function $\left(u_{0}(x)=1\right.$ for $x \leq 0$ and $u_{0}(x)=0$ for $x>0$ ), converges to the traveling wave with minimal speed $c^{*}=2 \sqrt{d}$.

In the framework introduced by Stokes (see the Introduction and Stokes, 1976), all these waves are pulled. Our results in Garnier et al. (2012) and Roques et al. (2012) prove that these waves are indeed pulled in the sense of Definition 1.1:

Theorem 2.1. If $f(u)=u(1-u)$ in equation (2.7), all the traveling waves $u(t, x)=U(x-c t)$ with $c \geq c^{*}$ are pulled in the sense of Definition 1.1.

This shows that, for the classical Fisher-KPP model, the diversity tends to decline during the colonization process, in the sense that every component except the rightmost tends to go extinct in the colonization front. Further results, for more general KPP nonlinearities are described in (Garnier et al., 2012), showing that the waves are pulled in any case.

This result is illustrated by the numerical simulations of Fig. 1. We have considered the case of a wave made of a finite number of components $\mu^{i}$ where $i=1, \ldots, N=7$. We assumed that these components were defined at $t=0$ by $\mu_{0}^{1}=U_{c} \cdot \mathbb{1}_{\left(-\infty, x_{1}\right]}, \mu_{0}^{k}=U_{c} \cdot \mathbb{1}_{\left(x_{k-1}, x_{k}\right]}$ for $k=2, \ldots, N-1$ and $\mu_{0}^{N}=U_{c} \cdot \mathbb{1}_{\left(x_{N-1},+\infty\right)}$, and for a sequence $x_{1}<x_{2}<\ldots<x_{N-1}$ of evenly distributed points. The point $x_{N-1}$ corresponds to the beginning of the rightmost component, which is not compactly supported (blue component in Fig. 1). As predicted by Theorem 2.1, all the components but the rightmost (blue) one are unable to follow the wave. From formula (1.6), the rightmost component converges to the wave itself, meaning a strong erosion of diversity. 


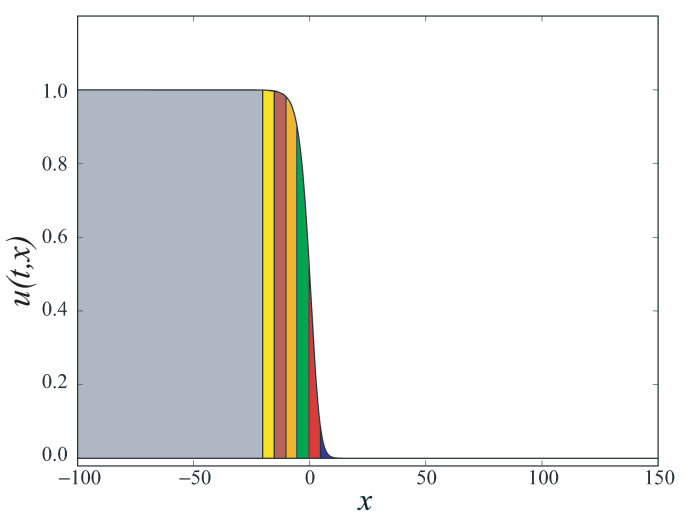

(a) $t=0$

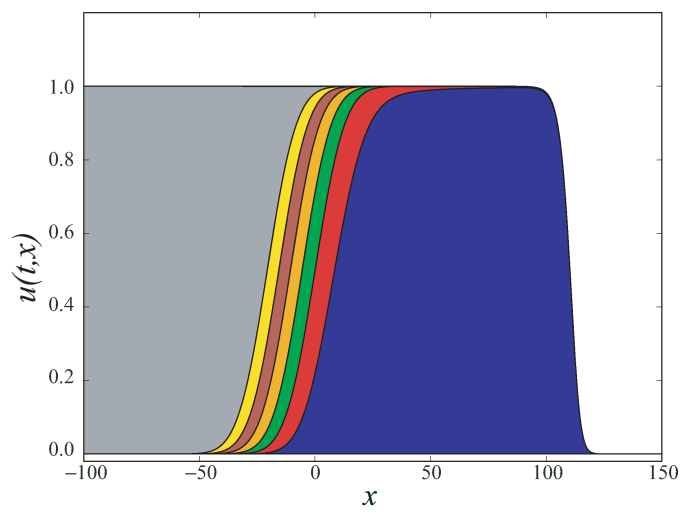

(b) $t=60$

Figure 1: Fisher-KPP case: dynamics of 7 components in the wave $u(t, x)=U(x-c t)$, with $d=1, f(u)=u(1-u)$ and $c=c^{*}=2$. Each component is depicted with a different color and with a thickness which corresponds, at each position $x$, to the density $\mu^{i}$ of the component.

\section{Role of the Allee effect}

A strong Allee effect corresponds to a negative growth rate at low densities, that is, $f(u)<0$ for $u \in(0, \rho)$, for some Allee threshold $\rho>0$. This might result from various factors: increased damage from bioagressors, increased mortality due to interspecific competition or reduced fitness due to suboptimal mating opportunities (Berec et al., 2007). This dynamic has been observed in many populations (Dennis, 1989; Veit and Lewis, 1996; Kramer et al., 2009). Moreover the Allee effect is known to affect the rate of spread of a population (Lewis and Kareiva, 1993; Barton and Turelli, 2011) and is expected to modify genetic structure on the edge of the population.

In this section, we again consider the equation (2.7) but with the classical bistable nonlinearity of the form:

$$
f(u)=u(1-u)(u-\rho),
$$

with $\rho \in(0,1 / 2)$.

The existence of a unique traveling wave solution $u(t, x)=U(x-c t)$ such that $U(-\infty)=1$ and $U(+\infty)=0$ has been proved in (Aronson and Weinberger, 1975; Fife and McLeod, 1977) for the growth functions (3.9). Furthermore, with $\rho \in(0,1 / 2)$, the speed $c$ is positive. Additionally, starting from a step-function $\left(u_{0}(x)=1\right.$ for $x \leq 0$ and $u_{0}(x)=0$ for $\left.x>0\right)$, the solution of the equation (2.7)-(3.9) converges to the unique traveling wave. Under the Allee assumption (3.9), the profile $U$ (up to shifts in $x$ ) and the speed $c$ of the traveling wave are explicitly known (see e.g. Petrovskii and Li, 2003):

$$
U(x)=\frac{1}{1+e^{x / \sqrt{2 d}}} \text { and } c=(1-2 \rho) \sqrt{\frac{d}{2}} .
$$

Garnier et al. (2012) and Roques et al. (2012) have shown that these waves are pushed:

Theorem 3.1. If $f(u)=u(1-u)(u-\rho)$ in equation (2.7), the unique traveling wave $u(t, x)=U(x-c t)$ is pushed in the sense of Definition 1.2. Furthermore, any component 
$\mu$ satisfying (1.5) with nonzero initial condition $\mu_{0}$, converges to a proportion $p$ of the wave $U$ uniformly on compact sets, in the moving frame with speed $c$ :

$$
\mu(t, x+c t) \rightarrow p U(x) \text { as } t \rightarrow+\infty, \text { uniformly on compact sets, }
$$

with

$$
p=p\left[\mu_{0}\right]=\frac{\int_{-\infty}^{+\infty} \mu_{0}(x) U(x) e^{\frac{c}{d} x} d x}{\int_{-\infty}^{+\infty} U^{2}(x) e^{\frac{c}{d} x} d x} \in(0,1] .
$$

The pushed nature of the wave shows that the Allee effect tends to promote the maintenance of genetic diversity in the wave. Moreover, the explicit description of $p\left[\mu_{0}\right]$ gives precise information on the origin of the individuals composing the wave at large times. Namely, let us consider the leftmost component defined by $\mu_{0}^{l}=U \cdot \mathbb{1}_{(-\infty, \alpha)}$ for $\alpha \in \mathbb{R}$. Differentiating $p(\alpha):=p\left[\mu_{0}^{l}\right]$ with respect to $\alpha$, we obtain a quantity $p^{\prime}(\alpha)$ that can be interpreted as the relative contribution to the wave of the individuals with an initial position $\alpha$. From the explicit formula (3.10) of the profile $U$, we can observe that $p^{\prime}$ reaches a unique maximum at the position

$$
\alpha_{\max }=\sqrt{2 d} \ln \left(\frac{1-2 \rho}{1+2 \rho}\right) .
$$

This formula emphasizes the advantageous role of the Allee effect to promote the components situated deep in the core of the population: the higher the Allee threshold $\rho$, the bigger the contribution of these individuals to the wave.

Further results, for more general bistable nonlinearities and for monostable nonlinearities corresponding to a weak Allee effect (that is when the per capita growth rate $f(u) / u$ is nonnegative but does not reach its maximum at $u=0$ ), are described in (Garnier et al., 2012). In the bistable case, the unique wave is always pushed while, in the monostable case, only the wave with minimal speed $c^{*}$ is pushed when the speed is nonlinear, i.e. when $c^{*}>2 \sqrt{d f^{\prime}(0)}$. These results are consistent with the terminology of Stokes (see Introduction).

Results of Theorem 3.1 are illustrated by numerical simulations presented in Fig. 2. Using the same framework as in Section 2, we observe in this case that, as predicted, all the components contribute to the advance of the wave.

\section{Interspecific competition: Lotka-Volterra reaction-diffusion systems}

Here, instead of colonizing an empty space, we consider a species $u$ invading a medium where a resident species $v$ is already present. We assume that both species are in competition. This scenario can be described using the following Lotka-Volterra system of reaction-diffusion equations (Murray, 2002):

$$
\left\{\begin{array}{l}
\partial_{t} u=d \partial_{x x} u+u\left(1-u-a_{1} v\right), \\
\partial_{t} v=\partial_{x x} v+r v\left(1-a_{2} u-v\right),
\end{array} \quad t>0, x \in \mathbb{R},\right.
$$

where all the coefficients $d, r, a_{1}, a_{2}$ are positive. The coefficients $a_{1}, a_{2}$ correspond to the interspecific competition coefficients. From a biological perspective, we assume competitive 


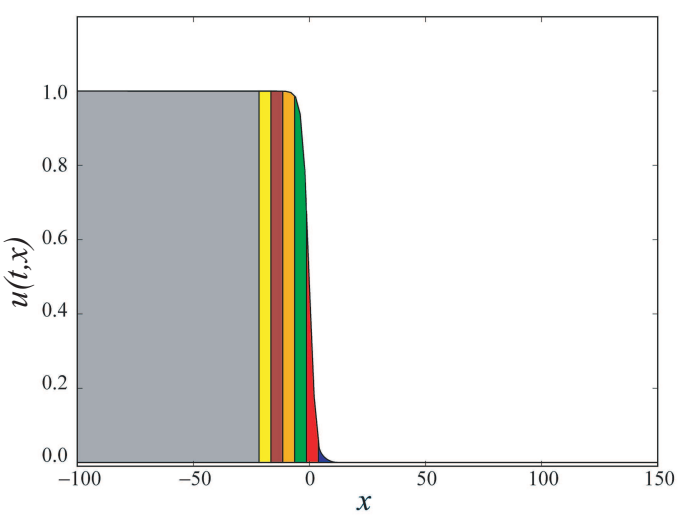

(a) $t=0$

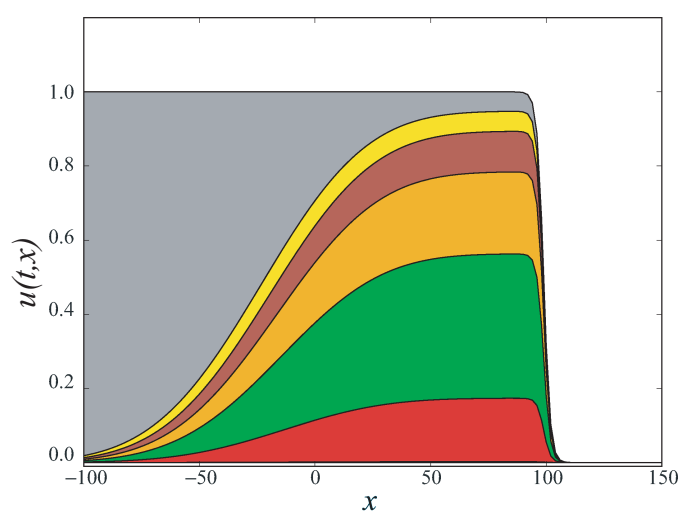

(b) $t=700$

Figure 2: Strong Allee effect : dynamics of seven components in the wave $u(t, x)=U(x-c t)$ satisfying (2.7) with $f(u)=u(1-u)(u-\rho)$, and $d=1, \rho=0.4$.

exclusion of the species $v$ by the species $u$. In other words, the species $u$ is a better competitor than $v$, in the sense that

$$
0<a_{1}<1<a_{2} .
$$

This assumption guarantees the existence of traveling waves which describe the invasion of the state $(0,1)$, where the species $u$ is not present, by the state $(1,0)$, where the species $v$ has been excluded. More precisely, in this context, the traveling wave solutions of speed $c>0$ are of the type $u(t, x)=U(x-c t)$, and $v(t, x)=V(x-c t)$, with limiting conditions:

$$
(U, V)(-\infty)=(1,0) \text { and }(U, V)(+\infty)=(0,1) .
$$

In this case, Kan-On (1997) has proved the existence of positive and monotone traveling waves if and only if $c \geq c^{*}>0$. The minimal speed $c^{*}$ is not known explicitly but Kan-On (1997) has given the following estimates:

$$
2 \sqrt{d\left(1-a_{1}\right)} \leq c^{*} \leq 2 \sqrt{d} .
$$

Note that, in the case $a_{1}=0$, the density $u$ of the invasive species follows the classical FisherKPP equation (2.7)-(2.8) studied in Section 2.

Depending on the parameters, the speed $c^{*}$ can be either linearly determined, that is $c^{*}=$ $2 \sqrt{d\left(1-a_{1}\right)}$, or nonlinearly determined if $c^{*}>2 \sqrt{d\left(1-a_{1}\right)}$. Sufficient conditions for the linear determinacy of the speed have been given by Lewis et al. (2002) and Huang (2010). Inspired by the results in the scalar case $a_{1}=0$ Murray (2002) and Okubo et al. (1989) conjectured that the speed $c^{*}$ was always linearly determined. Using asymptotic matching techniques, Hosono (2003) and Holzer and Scheel (2012) found that, in fact, the linear determinacy did not always hold true, especially for small values of $d$. Another recent example of nonlinear determinacy of the speed $c^{*}$ has been given by Huang and Han (2011). In the particular case $r=1 / d$, their result shows that, if $a_{1}$ is close enough to 1 , then $c^{*}>2 \sqrt{d\left(1-a_{1}\right)}$.

The linear determinacy of speed $c^{*}$ actually plays an important role on the inside dynamics of the wave $u(t, x)=U\left(x-c^{*} t\right)$ (Roques et al., 2013). For the system (4.13), the dynamics of 
any component $\mu$ in the wave $U$ is described by the following equation:

$$
\left\{\begin{aligned}
\partial_{t} \mu & =d \partial_{x x} \mu+\mu\left(1-u-a_{1} v\right), & & t>0, x \in \mathbb{R}, \\
\mu(0, x) & =\mu_{0}(x), & & x \in \mathbb{R},
\end{aligned}\right.
$$

We consider first the case where the speed $c^{*}$ is linearly determined:

Theorem 4.1. If $c^{*}=2 \sqrt{d\left(1-a_{1}\right)}$, then the wave $u(t, x)=U\left(x-c^{*} t\right)$ is a pulled wave in the sense of Definition 1.1.

This result, proved in (Roques et al., 2013), shows that, whenever the speed $c^{*}$ is linearly determined, the inside dynamics of the wave $u(t, x)=U\left(x-c^{*} t\right)$ is the same as in the FisherKPP case without competition. This means that the existence of a competitor for the invasive species has no effect on the erosion of its diversity.

We then consider the case of nonlinearly determined speeds $c^{*}$. In the previous sections, as well as in the monostable cases (see Garnier et al., 2012), the pulled/pushed nature of the waves turns out to be determined by the decay rate of the leading edge of the waves, as $x \rightarrow+\infty$ (see Remark 4.5 below). Thus, we distinguish two types of waves, depending on their exponential decay at $+\infty$.

Definition 4.2 (Slow-decay wave). A positive wave $u(t, x)=U(x-c t)$ with speed $c$ and such that $U(+\infty)=0$ is said to be a slow-decay wave if $\ln [U(y)] \sim-\lambda y$ as $y \rightarrow+\infty$, for some $0<\lambda \leq c /(2 d)$.

Definition 4.3 (Fast-decay wave). A positive wave $u(t, x)=U(x-c t)$ with speed $c$ and such that $U(+\infty)=0$ is said to be a fast-decay wave if $\ln [U(y)] \sim-\lambda y$ as $y \rightarrow+\infty$, for some $\lambda>c /(2 d)$.

Coming back to the system (4.13), it is known that the wave with minimal speed $c^{*}$ is a slow-decay wave if the speed is linearly determined (Morita and Tachibana, 2009). On the other hand, if the speed is nonlinearly determined, it is still unknown whether the wave with speed $c^{*}$ is a fast-decay or a slow-decay wave. The next result describes the inside dynamics of the wave $u(t, x)=U\left(x-c^{*} t\right)$ with nonlinearly determined speed $c^{*}$, in terms of its fast/slow decay nature.

Theorem 4.4. Assume that $c^{*}$ is nonlinearly determined:

$$
c^{*}>2 \sqrt{d\left(1-a_{1}\right)} .
$$

1) If $u(t, x)=U\left(x-c^{*} t\right)$ is a fast-decay wave, then it is a pushed wave in the sense of Definition 1.2. Furthermore, any component $\mu$ satisfying (4.16) with nonzero initial condition $\mu_{0}$, converges to a proportion $p$ of the wave $U$ uniformly in $\mathbb{R}$, in the moving frame with speed $c$ :

$$
\mu(t, x+c t) \rightarrow p\left[\mu_{0}\right] U(x) \text { uniformly in } \mathbb{R} \text { as } t \rightarrow+\infty,
$$

where $p\left[\mu_{0}\right]$ is given by $(3.11)$.

2) If $u(t, x)=U\left(x-c^{*} t\right)$ is a slow-decay wave, then it is a pulled wave in the sense of Definition 1.1. 


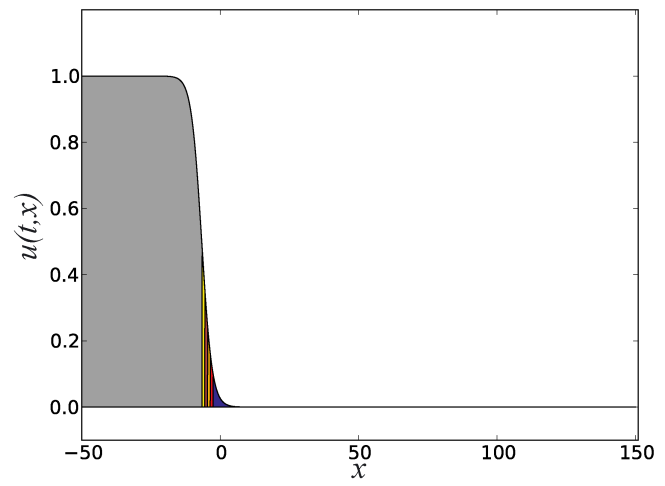

(a) $t=0$

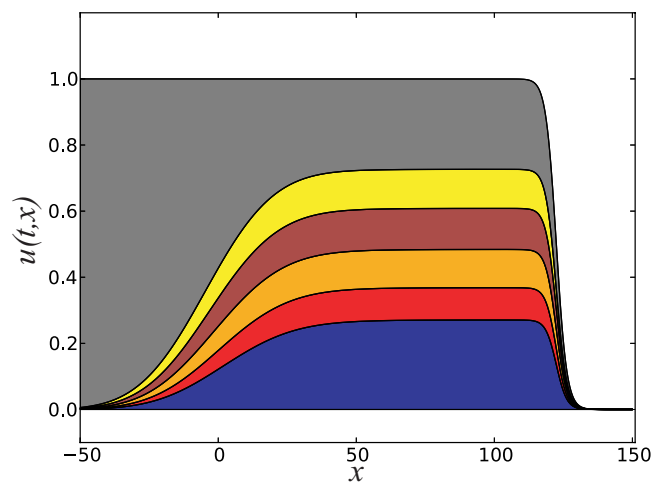

(b) $t=175$

Figure 3: Inside dynamics of the wave $u(t, x)=U\left(x-c^{*} t\right)$ in the presence of a competitor (system (4.13)). The parameter values are $r=d=1, a_{2}=2$ and $a_{1}=0.9$. The speed is nonlinear: $c^{*} \simeq 0.73>2 \sqrt{d\left(1-a_{1}\right)} \simeq 0.63$.

Based on numerical computations, Roques et al. (2013) have conjectured that traveling waves, with nonlinearly determined speed $c^{*}$, are always fast-decay waves as in the scalar case (see also Crooks, 2003). In particular, the waves described by Holzer and Scheel (2012) (for $0<d<<1$ ) and by Huang and Han (2011) (for $a_{1}$ close to 1), turn out to have a fast-decay; see numerical computations in (Roques et al., 2013) and remark 2 in (Holzer and Scheel, 2012). The numerical simulations of Fig. 3 confirm that the wave is pushed in the sense of Definition 1.2. This shows that competition with another species can lead to a better maintenance of diversity, compared to the case without competition $\left(a_{1}=0\right)$.

Remark 4.5. In the scalar case of equation (2.7), if $u(t, x)=U(x-c t)$ is a fast-decay wave, then the integral in the denominator of (3.11) converges, whereas, if it is a slow-decay wave with $\lambda<c /(2 d)$, then this integral diverges. It is known that in the Fisher-KPP case of Section 2, all the waves have a slow decay (Aronson and Weinberger, 1978), while in the strong Allee case of Section 3, the unique wave has a fast decay (see formula (3.10)). In the more general monostable case corresponding to a weak Allee effect, both cases could occur depending on $f(u)$. It turns out that slow-decay waves are pulled while fast-decay waves are pushed (Garnier et al., 2012). The analysis of the decay rates of the waves was one of the reasons for the introduction of pulled and pushed waves by Stokes (1976).

\section{Effect of a non-reproductive juvenile stage: delayed-differential equations}

In this section, we come back to the scalar equation:

$$
\partial_{t} u=d \partial_{x x} u+\mathcal{F}[u], t>0, x \in \mathbb{R},
$$

but with a nonlocal growth term $\mathcal{F}[u]=\mathcal{F}(u(t-\tau, x), u(t, x))$, with $\tau>0$. More precisely, we consider the Kobayashi's nonlinearity (Kobayashi, 1977):

$$
\mathcal{F}(u(t-\tau, x), u(t, x))=u(t-\tau, x)(1-u(t, x)) .
$$


In our ecological context, this nonlinearity represents a reproduction with a non-reproductive and motionless juvenile stage. During the life cycle of many insects, the juvenile stage consists of the egg, larva and pupa which cannot reproduce and do not move or move slowly compared to adults. In such cases, if the duration of the juvenile stage is $\tau$, the value of the growth term at time $t$ and position $x$ depends on the number of eggs which were laid at time $t-\tau$ at the position $x$. This number is a function of the density of adult individuals $u(t-\tau, x)$. The growth term can also depend on the current density of adult individuals, $u(t, x)$, because of crowding effects.

In the ecological literature, the existence of such a juvenile stage has been suspected to lead to a better conservation of the genetic diversity (Austerlitz et al., 2000). The existence of a delay in (5.18) is also well known to reduce the minimal speed of traveling waves for KPP-type nonlinearities (Schaaf, 1987). Indeed, positive traveling wave solutions $u(t, x)=U(x-c t)$ of equation (5.18)-(5.19) such that $U(-\infty)=1$ and $U(+\infty)=0$ exist if and only if $c \geq c^{*}(\tau)>0$, where $c^{*}(\tau)$ is decreasing with respect to $\tau>0$ (Schaaf, 1987); the case $c=c^{*}(\tau)$ can be treated as in Theorem 4.6 of Pan (2009).

Thus, as for the Allee effect, we could expect that the introduction of a delay term will modify the inside structure of the waves compared to the Fisher-KPP case with no delay considered in Section 2. This is actually not the case, since Bonnefon et al. (2013) have shown that all the waves with speeds $c>c^{*}(\tau)$ are pulled (with $\mu \mathcal{F}[u] / u(t, x)=\mu(t, x)(1-u(t, x)$ ) in $(1.5))$ :

Theorem 5.1. Any traveling wave $u(t, x)=U(x-c t)$ solving (5.18)-(5.19) with $c>c^{*}(\tau)$ is pulled in the sense of Definition 1.1.

This result is quite counter-intuitive regarding Sections 3 and 4. Indeed, the lower speed induced by the delay could have been advantageous for the components situated in the bulk of the population, as in the case with a strong Allee effect (Section 3). Besides, in the presence of a delay $\tau>0$, we have shown that, surprisingly, fast-decay waves can be pulled. This is a consequence of the following result (see Bonnefon et al., 2013):

Lemma 5.2. There exists a real number $\bar{c}(\tau) \in\left(c^{*}(\tau),+\infty\right)$ such that the waves with speeds $c \in\left(c^{*}(\tau), \bar{c}(\tau)\right)$ are fast-decay waves in the sense of Definition 4.3 and the waves with speeds $c \geq \bar{c}(\tau)$ are slow-decay waves in the sense of Definition 4.2.

Thus, fast-decay waves are not necessarily pushed. On the other hand, in all of the cases considered in Sections 2-5, the traveling waves with linearly determined speeds are pulled in the sense of Definition 1.1, and the traveling waves with nonlinearly determined speeds are pushed in the sense of Definition 1.2. A natural open question is whether or not this is true in general for reaction-diffusion models.

\section{Role of climate change}

Range shift processes are very frequent during climate changes (see e.g. Breed et al., 2013; Gayathri Samarasekera et al., 2012; Pluess, 2011; Rousselet et al., 2010). They are usually the combined effect of the colonization of newly emerging suitable habitat coinciding with the migration and then extinction in areas that have become unsuitable (Parmesan, 1996, 2006; Root et al., 2003; Walther et al., 2002). These series of population expansions and contractions have an important effect on genetic diversity (Davis and Shaw, 2001; Hewitt, 2000). Simulation 
studies have already pointed out that neutral genetic diversity of populations decreases with increased short-term regional climate variation (Cobben et al., 2011), with increased speed of the range shift (Arenas et al., 2012) or with decreased habitat suitability (McInerny et al., 2009). The aim of this section is to provide mathematical insights on the dynamics of neutral genetic diversity of a species under climate changes.

Following the framework provided in (Berestycki and Rossi, 2008; Berestycki et al., 2009; Potapov and Lewis, 2004), we assume that the environment is composed of one climate envelope of size $L>0$, surrounded by unfavorable habitat where population faces death at a constant rate. Due to climate change, the advantageous patch is shifted with a fixed speed $c \geq 0$. Thus, we consider the scalar equation (5.18) with $\mathcal{F}[u](t, x)=f(x-c t, u(t, x))$ :

$$
\partial_{t} u=d \partial_{x x} u+f(x-c t, u), t>0, x \in \mathbb{R},
$$

with the function $f$ defined by:

$$
f(x, u)= \begin{cases}u(1-u) & \text { if } x \in(0, L), \\ -u & \text { if } x \in(-\infty, 0] \cup[L,+\infty) .\end{cases}
$$

Thus, for any $d>0$, if the speed of the climate change $c$ is smaller than the spreading speed $c^{*}=2 \sqrt{d}$ in the homogeneous environment and if the size $L$ of the favorable patch is bigger than a critical size $L^{*}(d, c)$ then any solution $u$ of $(6.20)$ converges to a positive traveling wave solution of the form $u(t, x):=U(x-c t)$ with $U( \pm \infty)=0$ (Berestycki et al., 2009). The next result shows that this wave is always pushed (Garnier and Lewis, 2014).

Theorem 6.1. Assume that $0<c<c^{*}=2 \sqrt{d}$ and that $L>L^{*}(d, c)$. Then, the unique traveling wave $u(t, x)=U(x-c t)$ satisfying (6.20) is pushed in the sense of Definition 1.2. Furthermore, any component $\mu$ satisfying (1.5) with nonzero initial condition $\mu_{0}$, converges to a proportion $p$ of the wave $U$ uniformly in $\mathbb{R}$, in the moving frame with speed $c$ :

$$
\mu(t, x+c t) \rightarrow p\left[\mu_{0}\right] U(x) \text { uniformly in } \mathbb{R} \text { as } t \rightarrow+\infty,
$$

where $p\left[\mu_{0}\right]$ is given by (3.11).

Thus, as in the case of a strong Allee effect (Section 3) or in the case of a strong competition (Section 4), the detrimental effect of the environment outside the bounded region of size $L$ slows down the propagation and enables all the components to follow the wave. This leads to a higher genetic diversity compared to the classical Fisher-KPP case treated in Section 2. This is illustrated by the numerical simulations presented in Fig. 4.

\section{Effect of long-distance-dispersal: integro-differential equa- tions}

In ecology, long distance dispersal events are suspected to deeply modify the dynamics of a population (Bohrer et al., 2005; Cain et al., 2000; Nathan and Muller-Landau, 2000) and the genetic diversity in a population (Austerlitz and Garnier-Géré, 2003; Kot et al., 1996; Pringle et al., 2009). A classical example is the Reid's paradox of rapid plant migration (Reid, 1899; Roques et al., 2010). This rapid propagation phenomenon is usually explained using integrodifferential equations with fat-tailed kernels (Clark, 1998; Clark et al., 1998; Garnier, 2011; Kot et al., 1996; Medlock and Kot, 2003; Skellam, 1951) for which it is known that the positive 


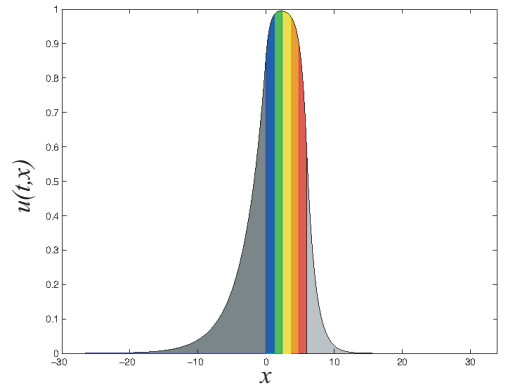

(a) $t=0$

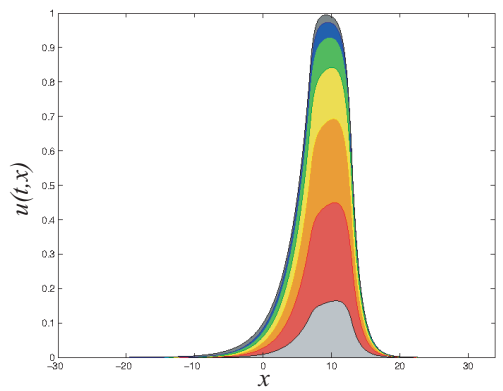

(b) $t=3.5$

Figure 4: Inside dynamics of the wave $u(t, x)=U(x-c t)$ in the presence of a shifting climate (equation (6.20)). The parameter values are $c=2, d=4$ and $L=6$.

solutions accelerate (Garnier, 2011; Kot et al., 1996; Medlock and Kot, 2003). These models are of the general form (1.1), with:

$$
\mathcal{D}[u](t, x)=(J \star u)(t, x)-u(t, x):=\int_{\mathbb{R}} J(x-y)(u(t, y)-u(t, x)) d y .
$$

The non-local term $J(x-y)$ represents the probability of moving from position $y$ to position $x$. The function $J$ is called the dispersal kernel. Throughout this section, we assume that the dispersal kernel $J: \mathbb{R} \rightarrow \mathbb{R}$ is a nonnegative even function of mass one that is

$$
J \in \mathcal{C}^{0}(\mathbb{R}), \quad J \geq 0, \quad J(x)=J(-x), \quad \text { and } \quad \int_{\mathbb{R}} J(x) d x=1 .
$$

One of the most interesting features of the nonlocal dispersion, compared to the classical diffusion, is that it can take into account rare long distance dispersal events. The frequency of occurrence of long distance dispersal events is encoded in the tail of the dispersal kernel. In the literature, two kinds of kernels can be distinguished: slowly decaying dispersal kernels (or fat-tailed kernels), and fast decaying (or thin-tailed) kernels.

Definition 7.1 (Thin-tailed dispersal kernels). The dispersal kernel $J$ is a thin-tailed kernel if

$$
\text { there exists } \lambda>0 \text {, such that } \int_{\mathbb{R}} J(x) e^{\lambda x} d x<\infty \text {. }
$$

This is the most common assumption among the mathematical literature on integrodifferential equations (see Aronson, 1977; Coville and Dupaigne, 2007; Diekmann, 1979; Thieme, 1979; Weinberger, 1982, 2002). This condition roughly means that $J$ decays at least like an exponential as $|x| \rightarrow+\infty$.

Definition 7.2 (Fat-tailed dispersal kernels). The dispersal kernel $J$ is a fat-tailed kernel if

$$
\text { for all } \eta>0 \text {, there exists } x_{\eta} \in \mathbb{R} \text { such that } J(x) \geq e^{-\eta x} \text { in }\left[x_{\eta},+\infty\right) \text {. }
$$

This assumption is equivalent to $J(x) e^{\eta|x|} \rightarrow \infty$ as $|x| \rightarrow \infty$ for all $\eta>0$ which means that $J$ decays slower than any exponentially decaying function.

Regarding the growth term, we assume in this section, as in Section 2, that

$$
\mathcal{F}[u]=f(u)=u(1-u) .
$$


The case of thin-tailed kernels. When the dispersal kernel $J$ is thin-tailed, the equation:

$$
\partial_{t} u=J \star u-u+u(1-u), t>0, x \in \mathbb{R},
$$

admits positive traveling waves solutions $u(t, x)=U(x-c t)$ such that $U(-\infty)=1$ and $U(+\infty)=0$ if and only if $c \geq c^{*}$, for some $c^{*}=c^{*}(J)>0$ (Carr and Chmaj, 2004; Coville and Dupaigne, 2007; Schumacher, 1980). The next result describes the inside dynamics of these waves (Bonnefon et al., 2014).

Theorem 7.3. If $J$ is a thin-tailed kernel, all traveling waves $u(t, x)=U(x-c t)$ satisfying (7.26) with $c \geq c^{*}$ are pulled in the sense of Definition 1.1.

Thus, although the dispersal term in model (7.26) is nonlocal, the inside dynamics of the traveling wave solutions is similar to that of the waves in the local reaction-diffusion model (2.7). In other words, when the kernel $J$ decays sufficiently fast at infinity, we observe a strong erosion of diversity during the colonization.

The case of fat-tailed kernels. When the dispersal kernel $J$ is fat-tailed, the equation (7.26) does not have traveling wave solutions (Yagisita, 2009). As shown by Garnier (2011), fat-tailed kernels lead to accelerating solutions which tend to flatten as they propagate. In order to be able to describe the inside dynamics of such solutions, and more generally of solutions which are not necessarily transition waves in the sense of Berestycki and Hamel (2012), we propose an extension of the framework described in the previous sections, and new notions of pulled and pushed solutions. The following definitions have been introduced in (Bonnefon et al., 2014).

Since $u$ ranges from 0 to 1 , we can define, for any level $\lambda \in(0,1)$ and $t>0$, the level set $E_{\lambda}(t)$ by

$$
E_{\lambda}(t)=\{x \in \mathbb{R}, u(t, x)=\lambda\} .
$$

Then, for any initial condition $u_{0}$ ranging in $[0,1]$, we denote by $\operatorname{Supp}\left(u_{0}\right)$ the support of $u_{0}$, that is the set $\operatorname{Supp}\left(u_{0}\right):=\overline{\left\{x \in \mathbb{R} \text { such that } u_{0}(x)>0\right\}}$, and we define $x_{0}^{-}:=\inf \left(\operatorname{Supp}\left(u_{0}\right)\right)$ and $x_{0}^{+}:=\sup \left(\operatorname{Supp}\left(u_{0}\right)\right)$. Note that if follows from the comparison principle that, as soon as $u_{0}$ has a nonzero integral, the solution $u$ of $(7.26)$ with initial condition $u_{0}$ is positive everywhere in $\mathbb{R}$ for every positive $t>0$ and the solutions $\mu^{i}$ of (1.3) are therefore well defined.

The notion of pulled and pushed solutions are defined as follows:

Definition 7.4 (Pulled solution to the right). A positive solution $u(t, x)$ of (1.1) starting from $u_{0}$ is said to be pulled to the right if for any component $\mu^{i}$ satisfying $(1.3), 0 \leq \mu_{0}^{i} \leq u(0, \cdot)$, $\mu_{0}^{i}(x)=0$ for large $x$ and $\overline{\operatorname{Supp}\left(\mu_{0}^{i}\right)} \subset\left[x_{0}^{-}, x_{0}^{+}\right)$, there holds

$$
\sup _{x \in E_{\lambda}^{+}(t)} \mu^{i}(t, x) \rightarrow 0, \text { as } t \rightarrow+\infty, \quad \text { for any level } \lambda \in(0,1) \text {, }
$$

where $E_{\lambda}^{+}(t):=E_{\lambda}(t) \cap(0,+\infty)$.

Conversely, we can define the notion of pushed solution to the right by

Definition 7.5 (Pushed solution to the right). A solution $u(t, x)$ of (1.1) starting from $u_{0}$, is said to be pushed to the right if there exists a component $\mu^{i}$ satisfying $(1.3), 0 \leq \mu_{0}^{i} \leq u(0, \cdot)$ and $\overline{\operatorname{Supp}\left(\mu_{0}^{i}\right)} \subset\left[x_{0}^{-}, x_{0}^{+}\right)$, and there exists a level $\lambda \in(0,1)$ such that

$$
\limsup _{t \rightarrow+\infty} \sup _{x \in E_{\lambda}^{+}(t)} \mu^{i}(t, x)>0,
$$


where $E_{\lambda}^{+}(t):=E_{\lambda}(t) \cap(0,+\infty)$.

We can easily define the notions of pushed and pulled solutions to the left. We just need to replace $\left[x_{0}^{-}, x_{0}^{+}\right)$by $\left(x_{0}^{-}, x_{0}^{+}\right]$and the positive part of the level set $E_{\lambda}^{+}(t)$ by the negative part $E_{\lambda}^{-}(t)$ defined by $E_{\lambda}^{-}(t):=E_{\lambda}(t) \cap(-\infty, 0)$, for any $\lambda \in(0,1)$ and $t>0$.

One can check that theses new definitions are consistent with the previous definitions 1.1 and 1.2 in the case of the inside dynamics of a traveling wave.

Using these definitions, we were able to describe in (Bonnefon et al., 2014) the inside dynamics of the solution of the Cauchy problem (7.26), starting with an initial condition $u_{0}$ made of two components $\mu_{0}^{1}$ and $\mu_{0}^{2}$, which are initially symmetric with respect to $x=0$, namely:

$$
\mu_{0}^{1}(x)=\mathbb{1}_{[-l, 0)} \text { and } \mu_{0}^{2}(x)=\mathbb{1}_{[0, l]},
$$

for some $l>0$. In (Bonnefon et al., 2014), a particular case of fat-tailed dispersal kernel was considered. This kernel corresponds to the Cauchy distribution:

$$
J(x)=\frac{\beta}{\pi\left(\beta^{2}+x^{2}\right)} \text { for all } x \in \mathbb{R},
$$

for some $\beta>0$.

The next result shows that the solution $u(t, x)$ of $(7.26)$ with the Cauchy kernel (7.29) is pushed to the right in the sense of Definition 7.5. Similarly, it is pushed to the left and the solution $u$ is thus pushed in any direction.

Theorem 7.6. There exists a time $\tau>0$ and a constant $\alpha>0$ such that

$$
\frac{\mu^{1}(t, x)}{u(t, x)} \geq \alpha \text { for all } t \geq \tau \text { and } x \in \mathbb{R} .
$$

This result shows the advantageous effect of long-distance-dispersal on the preservation of the diversity during the propagation. We conjecture that a comparable result holds for more general fat-tailed kernels.

Numerical simulations. We have carried out numerical simulations for both thin-tailed and fat-tailed kernels. The results are presented in Fig. 5. In the thin-tailed case, as predicted, the inside dynamics is comparable to that of the Fisher-KPP local equation (2.7) presented in Fig. 1. We observe a striking difference in the case of the fat-tailed Cauchy kernel. Firstly, as above-mentioned, the solution accelerates and tends to flatten; secondly, all the components are present in the leading edge of the solution. Interestingly, the leftmost component, which rapidly vanishes in the classical Fisher-KPP local equation, is the largest contributor to the solution.

\section{Conclusion}

The notions of pulled and pushed solutions introduced by Garnier et al. (2012) and Roques et al. (2012) are based on a decomposition of the solution of a reaction-dispersal equation into several components. In the framework of population dynamics, this decomposition, which is inspired by a work of Hallatschek and Nelson (2008), is related to the spatio-temporal evolution of the genetic structure of a population. The pulled solutions describe a rapid erosion of neutral 

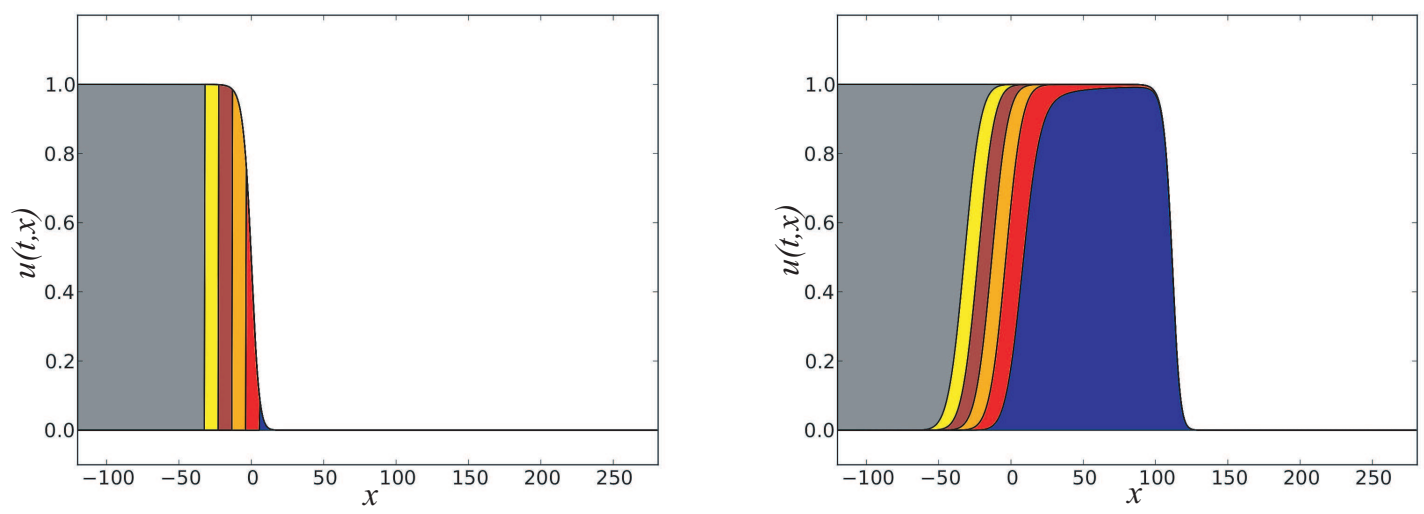

(a) Thin-tailed kernel
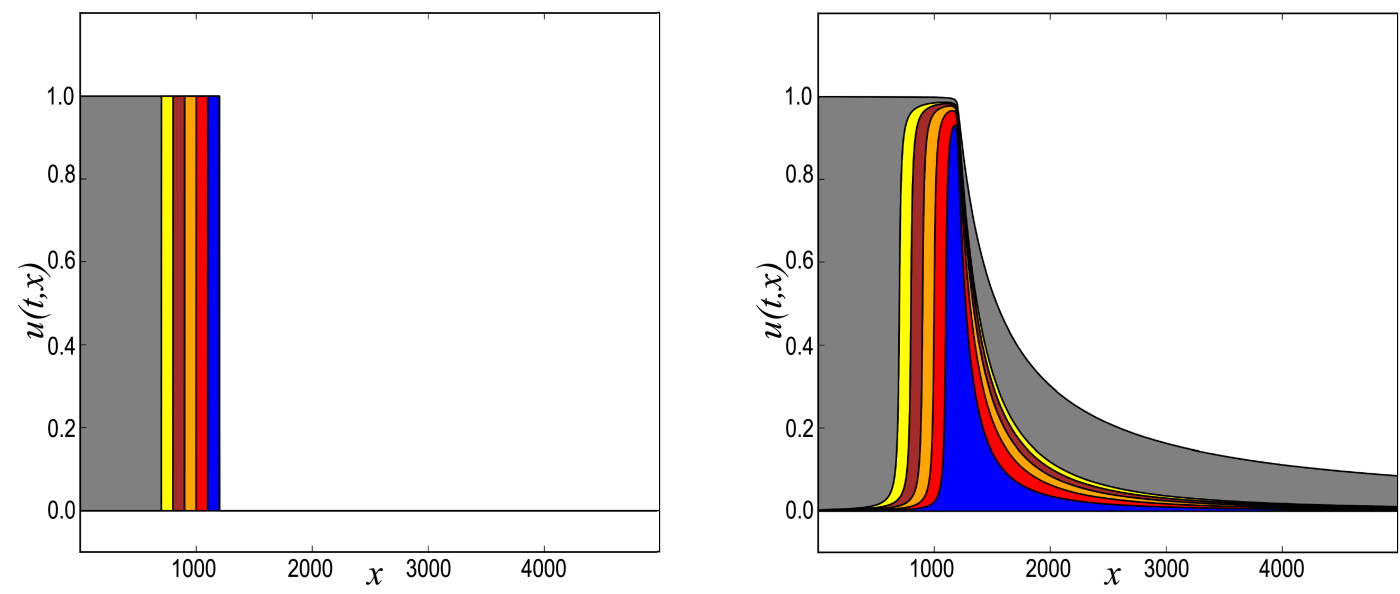

(b) Fat-tailed kernel

Figure 5: Inside dynamics of solutions of (7.26). (a): traveling wave solution in the case of the thin-tailed kernel $J(x)=(1 / 2) e^{-|x|}$, at $t=0$ (left) and $t=40$ (right). (b): solution starting from a step-function in the case of the Cauchy kernel (7.29) with $\beta=1$, at $t=0$ (left) and $t=6$ (right). 
genetic diversity due to the demographic advantage of isolated individuals ahead of the front, whereas the pushed solutions are associated with a maintenance of diversity. We present here a survey of the most recent applications of these notions to several standard models of population dynamics. We describe several counterintuitive results, where unfavorable factors for the persistence and spreading of a population tend to promote diversity in this population.

Section 2 focuses on the Fisher-KPP model. This is probably the most commonly used model of population dynamics (Kolmogorov et al., 1937; Skellam, 1951; Shigesada and Kawasaki, 1997; Turchin, 1998), and it often serves as a basis for the development of more complex models, such as those presented in the other sections of this paper. For this FisherKPP model, the traveling wave solutions are always pulled, which indicates a strong erosion of the diversity. Thus, any genetic component except the one most ahead of the wave vanishes progressively.

If we incorporate a strong Allee effect in the model, corresponding to a negative growth rate of the population at low densities, the inside dynamics of the solutions become totally different (Section 3). In this case, the traveling wave solutions are pushed, which means that all the genetic components of the population are conserved in the colonization wave at large times. Thus the richness of the population is preserved during the expansion but its evenness evolves since the proportion of the components differ depending on their initial condition. This result is in contrast with previous demographic studies on the Allee effect, which generally show adverse consequences of the Allee effect Garnier et al. (2012); Lewis and Kareiva (1993); Lewis and Van Den Driessche (1993). Here, because of the lower growth rate of the individuals at low densities, the components situated in the leading edge of the wave are penalized. This reduces the genetic drift due to founder effects, and enables the individuals situated in the core of the population to contribute to the colonization Roques et al. (2012).

In Sections 4 - 6, we analyzed other biological mechanisms which are suspected to play a similar role. Section 4 focuses on the effect of interspecific competition, by analyzing the inside dynamics of the solutions of Lotka-Volterra competition models. This corresponds to recent results obtained by Roques et al. (2013), which show that the existence of a competitor tends to promote the genetic diversity in an invasive population. Interestingly, no Allee effect is assumed here, but when the effect of competition becomes sufficiently strong, the traveling wave solutions switch from pulled waves to pushed waves. In that case, the advantageous growth rate of the low-density components situated in the leading edge of the wave is limited by the existence of a competitor. Again, this enables the genetic components situated in the core of the population to follow the colonization wave.

The recent results of Garnier and Lewis (2014), presented in Section 6, show that the existence of a climate constraint can have the same effect. Their result is based on the analysis of the inside dynamics of models with forced speed introduced in (Berestycki and Rossi, 2008; Berestycki et al., 2009; Potapov and Lewis, 2004). Again, no Allee effect was assumed in these models, and the reaction term is still of the Fisher-KPP type; however, it is assumed that the environment is unfavorable outside the climate envelope, which shifts with a constant speed. Because of this climate constraint, the individuals situated ahead of the wave are again penalized, since they are outside of the climate envelope. This leads to pushed solutions and therefore to a maintenance of diversity.

Contrarily to a previous study (Austerlitz et al., 2000), the results presented in Section 5, and which are based on the paper by Bonnefon et al. (2014), show that the presence of an immature and motionless juvenile stage does not have a significant effect on the genetic structure of an expanding population. One of the most interesting features of the inside dynamics of the 
waves in this case is that it does not depend on the decay rate of the wave, contrarily to the situations presented in Sections 2, 3, 4 and 6, where the pulled (resp. pushed) nature of the wave was determined by its slow (resp. fast) decay at infinity.

The results presented in Section 7 analyze the effect of the modality of dispersal on the neutral genetic diversity in a population. These results are based on the analysis of the inside dynamics of the solutions of integro-differential equations, with a dispersal kernel which can take long distance dispersal events into account. From a population genetics perspective, long distance dispersal events might have antagonistic consequences. They can either increase founder effects (Lambrinos, 2004) which can lead to an almost total loss of genetic diversity, or they can promote gene mixing on the leading edge of the wave of colonization (Klein et al., 2006). The effect of these events depends on the tail of the dispersal kernel $J$ which corresponds to the frequency at which the long distance dispersal events occur (Clark et al., 1998; Klein et al., 2006; Kot et al., 1996). A numerical study by Fayard et al. (2009) has shown that thin-tailed dispersal kernels tend to increase founder effects. In this case, only one gene will be represented on the leading edge of the colonization wave. This is an example where the genetic diversity is eroded during the expansion. On the other hand, the study of Fayard et al. (2009) shows that the genetic diversity tends to be preserved at the leading edge of the colonization wave when the dispersal kernel has a fat tail. The results that we have presented in Section 7 , and which are proved by Bonnefon et al. (2014), confirm these facts on a firm mathematical basis.

Related to our work is the notion a neutral mutation surfing. Several numerical studies have focused on this notion (Excoffier et al., 2009; Excoffier and Ray, 2008). Using a steppingstone model with a lattice structure, Edmonds et al. (2004) analyzed the fate of a neutral mutation present in the leading edge of an expanding population. Although in most cases the mutation remains at a low frequency in its original position, in some cases, surfing occurs when the mutation increases in frequency and propagates along the leading edge (Klopfstein et al., 2006). Using a backward-time numerical approach with stochastic demography, Hallatschek and Nelson (2008) provided an analysis of the surfing phenomenon in the presence of an Allee effect. They were able to connect their numerical findings to analytical formulas and concluded that surfing was not possible in deterministic reaction-diffusion, Fisher-KPP type models, i.e., without an Allee effect. In the forward-time deterministic approach presented here, the notion of surfing may not be appropriate and should be replaced by the more accurate notions of pulled and pushed waves. Surfing can indeed be associated with either (i) a rare gene becoming drastically dominant in the front or, (ii) with a gene initially present on the front being propagated alongside others present in the front. These two definitions lead to contradictory results, as with definition (i), gene surfing is only possible in pulled waves and for the furthest forward component of the wave, and definition (ii) dictates that gene surfing is promoted in pushed waves, since all the components initially present in the wave are able to surf.

The detailed proofs of the mathematical results presented in this paper can be found in the corresponding references. Let us just mention some general ideas and the main mathematical tools which have been used in these proofs. In all cases, the determination of the pushed nature of a wave $u(t, x)=U(x-c t)$ is based on the spectral properties of a Schrödinger operator

$$
\mathcal{L}=-d \partial_{x x}+\left(\frac{c^{2}}{4 d}-g(x, U)\right)
$$

where $g$ is the per capita growth rate of $u: g(x, U)=f(U(x)) / U(x)$ in Sections 3,6 and $g(x, U)=1-U(x)-a_{1} V(x)$ in Section 4. The fast-decay nature (see Definition 4.3) of a wave 
$U$ is a key ingredient in the proof of the pushed nature of the wave. In particular, it implies that $\varphi(x)=e^{\frac{c x}{2 d}} U(x)$, which is a positive eigenfunction of $\mathcal{L}$ with the associated eigenvalue 0 , belongs to $L^{2}(\mathbb{R})$. However, the results of Theorem 5.1 and Lemma 5.2 show that the fast-decay of a wave $u(t, x)=U(x-c t)$ is not sufficient in general to imply that this wave is pushed. When a delay $\tau$ is added in the reaction term $F(u(t-\tau, x), u(t, x))=u(t-\tau, x)(1-u(t, x))$, there exist slow-decay and fast-decay waves, but all of them are pulled. This can be proved by constructing an explicit exponential super-solution of the equation satisfied by a component $\mu$, and by using a maximum principle. A comparable method is used in the proof of the pulled nature of a wave for integro-differential equations with thin-tailed kernels and KPP reaction terms.

In the other cases described in this paper, proving that a wave is pulled bears on some integral estimates of the proportion $\mu / u$ in suitable weighted spaces. A key ingredient is again the asymptotic behavior of the wave $u(t, x)=U(x-c t)$, as $x \rightarrow+\infty$, since these integral estimates mainly rely on the slow-decay nature of the wave.

In the case of integro-differential equations with fat-tailed kernels, traveling wave solutions do not exist (Garnier, 2011; Yagisita, 2009). The proof of the pushed nature of the solutions (in the sense of Definition 7.5) is based on totally different arguments. This proof uses a couple of sub-solution and super-solution for the equation satisfied by a component $\mu$ of the solution, whose ratio remains bounded away from 0 . The fat-tailed nature of the kernel $J$ of course plays a crucial role. In particular, the proof is based on the fact that, for any $L>0, J(x+L) / J(x)$ is bounded from below by a positive constant, uniformly in $x \in \mathbb{R}$. This property is satisfied as soon as $\ln (J(x))$ decays linearly or slower as $x \rightarrow+\infty$; it is therefore verified by all fat-tailed kernels. This is an important feature of fat-tailed kernels, which has been first noticed by Klein et al. (2006).

Considering alleles under selection would be a natural extension of the results presented here. However, this would imply violating the neutrality assumptions on the components $\mu^{i}$ $(i \in I)$, which reduced the analysis of a $\operatorname{card}(I)+n$-dimensional system to the analysis of a system of $n+1$ equations, where $n$ is the number of equations governing the dynamics of the total solution $u(t, x)$ ( $n=2$ in Section 4 and $n=1$ in the other sections). Thus, considering nonneutral components under selection would lead to large competition systems. These systems are nonmonotone, and cannot be reduced to a monotone system with a simple change of variable when the number of components is larger than 2, which makes their mathematical analysis extremely complex.

\section{Acknowledgements}

The research leading to these results has received funding from the European Research Council under the European Union's Seventh Framework Programme (FP/2007-2013) / ERC Grant Agreement n.321186 - ReaDi - Reaction-Diffusion Equations, Propagation and Modelling, and from the French Agence Nationale pour la Recherche, within the ANR-10-INTB-1705-04MACBI project. This work has also been carried out in the framework of the Labex Archimède (ANR-11-LABX-0033) and of the A*MIDEX project (ANR-11-IDEX-0001-02), funded by the "Investissements d'Avenir" French Government program managed by the French National Research Agency (ANR). Lastly, we thank the two anonymous reviewers for their insightful comments. 


\section{References}

Arenas, M., N. Ray, M. Currat, and L. Excoffier (2012). Consequences of range contractions and range shifts on molecular diversity. Mol Biol Evol 29(1), 207-218.

Aronson, D. G. (1977). The asymptotic speed of propagation of a simple epidemic. In W. E. Fitzgibbon and H. F. Walker (Eds.), Nonlinear Diffusion, pp. 1-23. Pitman, London.

Aronson, D. G. and H. G. Weinberger (1975). Nonlinear diffusion in population genetics, combustion and nerve propagation. In Partial Differential Equations and Related Topics, Volume 446 of Lectures Notes Math, pp. 5-49. Springer, New York.

Aronson, D. G. and H. G. Weinberger (1978). Multidimensional nonlinear diffusion arising in population genetics. Adv Math 30(1), 33-76.

Austerlitz, F. and P. H. Garnier-Géré (2003). Modelling the impact of colonisation on genetic diversity and differentiation of forest trees: interaction of life cycle, pollen flow and seed long-distance dispersal. Heredity 90(4), 282-290.

Austerlitz, F., S. Mariette, N. Machon, P. H. Gouyon, and B. Godelle (2000). Effects of colonization processes on genetic diversity: differences between annual plants and tree species. Genetics 154(3), 1309-1321.

Barton, N. H. and M. Turelli (2011). Spatial waves of advance with bistable dynamics: cytoplasmic and genetic analogues of Allee effects. Am Nat 178(3), E48-E75.

Berec, L., E. Angulo, and F. Courchamp (2007). Multiple Allee effects and population management. Trends Ecol Evol 22, 185-191.

Berestycki, H., O. Diekmann, C. J. Nagelkerke, and P. A. Zegeling (2009). Can a species keep pace with a shifting climate? Bull Math Biol 71(2), 399-429.

Berestycki, H. and F. Hamel (2012). Generalized transition waves and their properties. Comm Pure Appl Math 65, 592-648.

Berestycki, H. and L. Rossi (2008). Reaction-diffusion equations for population dynamics with forced speed I - the case of the whole space. Disc Cont Dyn Systems A 21(1), 41-67.

Bohrer, G., R. Nathan, and S. Volis (2005). Effects of long-distance dispersal for metapopulation survival and genetic structure at ecological time and spatial scales. J. Ecol. 93(5), $1029-1040$.

Bonnefon, O., J. Coville, J. Garnier, and L. Roques (2014). Inside dynamics of solutions of integro-differential equations. Disc Cont Dyn Systems B, in press.

Bonnefon, O., J. Garnier, F. Hamel, and L. Roques (2013). Inside dynamics of delayed traveling waves. Math Model Nat Phenom 8, 44-61.

Breed, G., S. Stichter, and E. E. Crone (2013). Climate-driven changes in northeastern US butterfly communities. Nature Clim. Change 3, 142-145.

Cain, M., B. Milligan, and A. Strand (2000). Long distance seed dispersal in plant populations. Am. J. Bot. 87(9), 1217-1227. 
Carr, A. and J. Chmaj (2004). Uniqueness of travelling waves for nonlocal monostable equations. Proc. Amer. Math. Soc. 132(8), 2433-2439.

Clark, J. S. (1998). Why trees migrate so fast: Confronting theory with dispersal biology and the paleorecord. Am Nat 152, 204-224.

Clark, J. S., C. Fastie, G. Hurtt, S. T. Jackson, C. Johnson, G. King, M. Lewis, J. Lynch, S. Pacala, I. C. Prentice, E. W. Schupp, T. Webb III, and P. Wyckoff (1998). Reid's Paradox of rapid plant migration. BioScience 48, 13-24.

Cobben, M. M. P., J. Verboom, P. F. M. Opdam, R. F. Hoekstra, R. Jochem, P. Arens, and M. J. M. Smulders (2011). Projected climate change causes loss and redistribution of genetic diversity in a model metapopulation of a medium-good disperser. Ecography 34(6), 920-932.

Coville, J. and L. Dupaigne (2007). On a nonlocal reaction diffusion equation arising in population dynamics. Proc R Soc Edin A 137, 1-29.

Crooks, E. C. M. (2003). Travelling fronts for monostable reaction-diffusion systems with gradient-dependence. Advances Diff Equations 8, 279-314.

Davis, M. B. and R. G. Shaw (2001). Range shifts and adaptive responses to quaternary climate change. Science 292(5517), 673-679.

Dennis, B. (1989). Allee effects: population growth, critical density, and the chance of extinction. Natural Resource Modeling 3, 481-538.

Diekmann, O. (1979). Run for your life. a note on the asymptotic speed of propagation of an epidemic. J Diff Equations 33, 58-73.

Edmonds, C. A., A. S. Lillie, and L. L. Cavalli-Sforza (2004). Mutations arising in the wave front of an expanding population. Proc Natl Acad Sci USA 101(4), 975-979.

Excoffier, L., M. Foll, and R. J. Petit (2009). Genetic consequences of range expansions. Annu Rev Ecol Evol Syst 40(1), 481-501.

Excoffier, L. and N. Ray (2008). Surfing during population expansions promotes genetic revolutions and structuration. Trends Ecol Evol 23(7), 347-351.

Fayard, J., E. K. Klein, and F. Lefèvre (2009). Long distance dispersal and the fate of a gene from the colonization front. J Evol Biol 22(11), 2171-2182.

Fife, P. C. (1979). Mathematical Aspects of Reacting and Diffusing Systems, Volume 28 of Lecture Notes in Biomathematics. Springer-Verlag.

Fife, P. C. and J. McLeod (1977). The approach of solutions of nonlinear diffusion equations to traveling front solutions. Arch Ration Mech Anal 65(1), 335-361.

Fisher, R. A. (1937). The wave of advance of advantageous genes. Ann Eugen 7, 335-369.

Garnier, J. (2011). Accelerating solutions in integro-differential equations. SIAM J Math Anal 43, 1955-1974. 
Garnier, J., T. Giletti, F. Hamel, and L. Roques (2012). Inside dynamics of pulled and pushed fronts. J Math Pures Appl 98, 428-449.

Garnier, J. and M. A. Lewis (2014). Expansion under climate change: the genetic consequences. Preprint.

Garnier, J., L. Roques, and F. Hamel (2012). Success rate of a biological invasion in terms of the spatial distribution of the founding population. Bull Math Biol 74, 453-473.

Gayathri Samarasekera, G. D. N., N. V. Bartell, B. S. Lindgren, J. E. K. Cooke, C. S. Davis, P. M. A. James, D. W. Coltman, K. E. Mock, and B. W. Murray (2012). Spatial genetic structure of the mountain pine beetle (Dendroctonus ponderosae) outbreak in western Canada: historical patterns and contemporary dispersal. Mol Ecol 21, 2931-2948.

Hadeler, K. P. and F. Rothe (1975). Travelling fronts in nonlinear diffusion equations. J Math Biol 2(3), 251-263.

Hallatschek, O. and D. R. Nelson (2008). Gene surfing in expanding populations. Theor Popul Biol 73, 158-170.

Hewitt, G. M. (2000). The genetic legacy of the quarternary ice ages. Nature 405(22 June 2000), 907-913.

Holzer, M. and A. Scheel (2012). A slow pushed front in a Lotka-Volterra competition model. Nonlinearity 25(7), 2151.

Hosono, Y. (2003). Traveling waves for a diffusive Lotka-Volterra competition model I: singular perturbations. Disc Cont Dyn Systems B 3, 79-95.

Huang, W. (2010). Problem on minimum wave speed for a Lotka-Volterra reaction-diffusion competition model. J Dyn Differ Equ 22(2), 285-297.

Huang, W. and M. Han (2011). Non-linear determinacy of minimum wave speed for a LotkaVolterra competition model. J Diff Equations 251(6), 1549-1561.

Kan-On, Y. (1997). Fisher wave for the Lotka-Volterra competition model with diffusion. Nonlinear Anal-Theor 28, 145-164.

Klein, E. K., C. Lavigne, and P. H. Gouyon (2006). Mixing of propagules from discrete sources at long distance: comparing a dispersal tail to an exponential. BMC Ecology 6(3).

Klopfstein, S., M. Currat, and L. Excoffier (2006). The fate of mutations surfing on the wave of a range expansion. Mol Biol Evol 23(3), 482-490.

Kobayashi, K. (1977). On the semilinear heat equations with time-lag. Hiroshima Math $J$ \%, 459-472.

Kolmogorov, A. N., I. G. Petrovsky, and N. S. Piskunov (1937). Étude de l'équation de la diffusion avec croissance de la quantité de matière et son application à un problème biologique. Bull Univ État Moscou, Sér Int A 1, 1-26.

Kot, M., M. Lewis, and P. van den Driessche (1996). Dispersal data and the spread of invading organisms. Ecology 77, 2027-2042. 
Kramer, A. M., B. Dennis, A. M. Liebhold, and J. M. Drake (2009). The evidence for Allee effects. Popul Ecol 51, 341-354.

Lambrinos, J. G. (2004). How interactions between ecology and evolution influence contemporary invasion dynamics. Ecology 85(8), 2061-2070.

Lewis, M. A. and P. Kareiva (1993). Allee dynamics and the spread of invading organisms. Theor Popul Biol 43, 141-158.

Lewis, M. A., B. Li, and H. F. Weinberger (2002). Spreading speed and linear determinacy for two-species competition models. J Math Biol 45(3), 219-233.

Lewis, M. A. and P. Van Den Driessche (1993). Waves of extinction from sterile insect release. Math Biosci 116(2), 221-247.

McInerny, G. J., J. R. G. Turner, H. Y. Wong, J. M. J. Travis, and T. G. Benton (2009). How range shifts induced by climate change affect neutral evolution. Proc $R$ Soc B 276(1661), $1527-1534$.

Medlock, J. and M. Kot (2003). Spreading disease: Integro-differential equations old and new. Math Biosci 184(2), 201-222.

Morita, Y. and K. Tachibana (2009). An entire solution to the Lotka-Volterra competitiondiffusion equations. SIAM J Math Anal 40(6), 2217-2240.

Murray, J. D. (2002). Mathematical Biology. Third edition, Interdisciplinary Applied Mathematics 17, Springer-Verlag, New York.

Nathan, R. and H. Muller-Landau (2000). Spatial patterns of seed dispersal, their determinants and consequences for recruitment. Trends Ecol Evol 15(7), 278-285.

Okubo, A., P. K. Maini, M. H. Williamson, and J. D. Murray (1989). On the spatial spread of the grey squirrel in britain. Proc R Soc B. Biol Sci 238(1291), 113-125.

Pan, S. (2009). Asymptotic behavior of travelling fronts of the delayed Fisher equation. Nonlinear Anal Real World Appl 10, 1173-1182.

Parmesan, C. (1996). Climate and species' range. Nature 382, 765-766.

Parmesan, C. (2006). Evolutionary and ecological responses to recent climate change. Annu Rev Ecol Evol 37(8), 637-669.

Petrovskii, S. and B. Li (2003). An exactly solvable model of population dynamics with densitydependent migration and Allee effect. Math Biosci 186, 79-91.

Pluess, A. R. (2011). Pursuing glacier retreat: genetic structure of a rapidly expanding larix decidua population. Mol Ecol 20(3), 473-485.

Potapov, A. B. and M. A. Lewis (2004). Climate and competition: the effect of moving range boundaries on habitat invasibility. Bull Math Biol 66(5), 975-1008.

Pringle, J. M., F. Lutscher, and E. Glick (2009). Going against the flow: effects of non-Gaussian dispersal kernels and reproduction over multiple generations. Mar Ecol Prog Ser 377(1), 1317. 
Reid, C. (1899). The origin of the British flora. London : Dulau \& Co.

Root, T. L., J. T. Price, K. R. Hall, S. H. Schneider, C. Rosenzweig, and J. A. Pounds (2003). Fingerprints of global warming on wild animals and plants. Nature 421 (6918), 57-60.

Roques, L., J. Garnier, F. Hamel, and E. K. Klein (2012). Allee effect promotes diversity in traveling waves of colonization. Proc Natl Acad Sci USA 109(23), 8828-8833.

Roques, L., F. Hamel, J. Fayard, B. Fady, and E. K. Klein (2010). Recolonisation by diffusion can generate increasing rates of spread. Theor Popul Biol 77, 205-212.

Roques, L., Y. Hosono, O. Bonnefon, and T. Boivin (2013). The effect of competition on the neutral intraspecific diversity of invasive species. Preprint.

Rothe, F. (1981). Convergence to pushed fronts. Rocky Mountain Journal of Mathematics 11(4), 617-634.

Rousselet, J., R. Zhao, D. Argal, M. Simonato, A. Battisti, A. Roques, and C. Kerdelhué (2010). The role of topography in structuring the demographic history of the pine processionary moth, Thaumetopoea pityocampa (Lepidoptera: Notodontidae). J Biogeogr 37, 1478-1490.

Schaaf, K. W. (1987). Asymptotic behavior and traveling wave solutions for parabolic functional differential equations. Trans Amer Math Soc 302, 587-615.

Schumacher, K. (1980). Travelling-front solutions for integro-differential equations. I. J. Reine Angew. Math. 316, 54-70.

Shigesada, N. and K. Kawasaki (1997). Biological Invasions: Theory and Practice. Oxford Series in Ecology and Evolution, Oxford: Oxford University Press.

Skellam, J. G. (1951). Random dispersal in theoretical populations. Biometrika 38, 196-218.

Stokes, A. N. (1976). On two types of moving front in quasilinear diffusion. Math Biosci 31, 307-315.

Thieme, H. R. (1979). Density-dependent regulation of spatially distributed populations and their asymptotic speed of spread. J Math Biol 8, 173-187. 10.1007/BF00279720.

Turchin, P. (1998). Quantitative Analysis of Movement: Measuring and Modeling Population Redistribution in Animals and Plants. Sinauer, Sunderland, MA.

Veit, R. R. and M. A. Lewis (1996). Dispersal, population growth, and the Allee effect: dynamics of the house finch invasion of eastern North America. Am Nat 148, 255-274.

Walther, G.-R., E. Post, P. Convey, A. Menzel, C. Parmesan, T. Beebee, J.-M. Fromentin, O. Hoegh-Guldberg, and F. Bairlein (2002). Ecological responses to recent climate change. Nature 416(6879), 389-395.

Weinberger, H. F. (1982). Long-time behavior of a class of biological models. SIAM J Math Anal 13(3), 353-396.

Weinberger, H. F. (2002). On spreading speeds and traveling waves for growth and migration in periodic habitat. $J$ Math Biol 45, 511-548. 
Yagisita, H. (2009). Existence and nonexistence of travelling waves for a nonlocal monostable equation. Publ. RIMS, Kyoto Univ. 45, 925-953. 